Check for updates

Cite this: J. Mater. Chem. A, 2020, 8 , 22435

DOI: $10.1039 / d 0 \operatorname{ta} 90244 f$

rsc.li/materials-a

\section{Correction: Construction of a hierarchical carbon nanotube/MXene membrane with distinct fusiform channels for efficient molecular separation}

\author{
Mingmei Ding, ${ }^{\text {ab }}$ Hang Xu, ${ }^{\text {*a }}$ Wei Chen, ${ }^{a}$ Qing Kong, ${ }^{a}$ Tao Lin, ${ }^{a}$ Hui Tao, ${ }^{a}$ Kai Zhang, ${ }^{c}$
} Qin Liu, ${ }^{\text {bd }}$ Kaisong Zhang ${ }^{d}$ and Zongli Xie ${ }^{\star b}$

Correction for 'Construction of a hierarchical carbon nanotube/MXene membrane with distinct fusiform channels for efficient molecular separation' by Mingmei Ding et al., J. Mater. Chem. A, 2020, DOI: 10.1039/d0ta07354g.

The authors regret an error in the Acknowledgements section of the published article which meant that Jacinta White was not acknowledged correctly. The corrected Acknowledgements section should read as follows:

This work was financially supported by CSIRO Manufacturing, the Fundamental Research Funds for the Central Universities and the World-Class Universities (Disciplines), the Characteristic Development Guidance Funds for the Central Universities, the National Key Research and Development Project of China (2016YFC0400803), the National Natural Science Foundation of China (No. 51738013 and No. 51978239), the 111 Project under grant number B20044, and the Priority Academic Program Development Project of Jiangsu Higher Education Institutions (PAPD). Mingmei Ding and Qin Liu would like to acknowledge the scholarship received from the China Scholarship Council (CSC). The authors would like to acknowledge Ray Huang, Derrick Ng, Jacinta White, Aaron Seeber, and Mark Greaves from CSIRO for their assistance with water contact angle measurement, BET, TEM, XRD analyses, and SEM training, respectively. The authors would also like to thank Dr B. Chen and Prof. Q. Zhou from Nanjing Tech University for performing the AIMD simulations of the studied electrolyte using VASP.

The Royal Society of Chemistry apologises for these errors and any consequent inconvenience to authors and readers.

${ }^{a}$ Ministry of Education Key Laboratory of Integrated Regulation and Resource Development on Shallow Lakes, College of Environment, Hohai University, Nanjing 210098, China. E-mail: xuhang810826@163.com

${ }^{b}$ CSIRO Manufacturing, Private Bag 10, Clayton South, Vic. 3169, Australia. E-mail: zongli.xie@csiro.au

'State Key Laboratory of Environmental Aquatic Chemistry, Key Laboratory of Drinking Water Science and Technology, Research Center for Eco-Environmental Sciences, Chinese Academy of Sciences, Beijing, 100085, China

${ }^{d}$ Institute of Urban Environment, Chinese Academy of Sciences, Xiamen 361021, P. R. China 